\title{
Developing consciousness of health and motivating adolescent students to quit unhealthy habits.
}

\begin{abstract}
Sai Shri Ramamurthy
Sree Venkateshwara Pre University College, Madiwala, Bengaluru

\section{Abstract}

This paper talks about the survey conducted before and after the presentation on smoking cigarettes, reverse smoking and consuming alcohol. The target group was undergraduate students of the non science background from the Bangalore urban district. The lecturer handling non-core subjects like environmental science and science and technology conducted the survey of people with unhealthy habits such as smoking and drinking. A presentation was done by the lecturer about the ill-effects of the same and a survey was conducted after the presentation and a keen follow up was done and the response from the students were honest. The number of students who smoke and drink significantly reduced.
\end{abstract}

Keywords : Smoking, reverse smoking, alcohol, systemic lupus erythematosus, rheumatoid arthritis, Hairfall and ageing.

\section{Introduction :}

A group of undergraduate students from the Bangalore urban district was chosen for the study. The students were given a presentation about the ill effects of unhealthy habits. A survey was conducted after the presentation. Because the students were from the nonscience background a general introduction was given to the students about selected diseases and the aftermath of the same.

\section{Ill effects of smoking and drinking}

\section{Systemic Lupus Erythematosus}

SLE commonly called as lupus has smoking as one of its causal factors. And smoking in Lupus patients causes a disease flare. Current smoking rather than former smoking is a greater risk factor in SLE, Smoking is more common in patients with SLE than in population controls of similar age and socio-economic status. Current and former smoking is a risk factor for the development of SLE. SLE also causes hairloss and alopecia. 


\section{Rheumatoid arthritis}

Rheumatoid arthritis (RA) is a chronic inflammatory disease caused by both genetic and environmental factors. Smoking has been implicated as one of the most important extrinsic risk factors for its development and severity.

\section{Smoking and accelerated ageing}

Smoking makes women look twice as old as their age and men one and a half times older than women. Though smoking causes premature ageing cessation of smoking could possibly help reverse the effects.

\section{Alcohol and obesity}

Though the degree of obesity caused is proportional to the amount of intake of alcohol, consumption of alcohol has a positive relationship with obesity both in men and women. It also affects the sleep habits and eating habits.

\section{Effect of alcohol on endurance exercises}

Effect of alcohol in the metabolism of the body has a direct effect on the endurance levels of the athletes of aerobic and anaerobic sports.

\section{Effect of alcohol on the hormones and fertility}

Alcohol consumption has different types of effects on men and women. In men it affects the level of testosterone and the quality of sperms produced. In women it might aggravate Dysmenorrhea.

Alcohol intake during pregnancy could cause Foetal Alcoholic Syndrome Disorder affecting the physical and mental development of the foetus. Excessive alcohol intake in women might also cause disturbances in fertility. Moreover it can also intervene in the fertility treatments.

\section{Hypothesis:}

1. There was no significant difference in the survey conducted before the presentation and after the presentation.

2. There is a significant difference between the survey conducted before and after the presentation. 


\section{Methodology of the research}

A survey was conducted to find out the number of people who smoke and drink. The students were given an introduction about the course of the disease., the deformity that rheumatoid arthritis could cause and the effects of SLE. The damage unhealthy habits could cause to the genetic material and mutations in DNA. Though alcohol in minimal effects are often found to have beneficial effects it is also addictive in nature and alcohol in moderate and excessive amounts are found to cause diseases and permanent disorders.

\section{Results and discussions}

There was a significant difference between the number of people who smoke and drink significantly reduced after the presentation.

The problems that bothered the students were accelerated ageing, alopecia and the amount it would cost for the treatment of diseases like arthritis, SLE, cancer and also the effect it has on the endurance level. Moreover the fact that the cessation of these habits could possibly bring about a reversal of the adverse effects caused motivated the students

Before the presentation there were $33 \%$ of smokers and drinkers, after the survey though the entire group didn't quit smoking cigarettes and drinking the percentage of population of smokers and drinkers significantly reduced to $16 \%$ and all the adolescent girls in the smoking and drinking population accepted to quit the unhealthy habits.

\section{Limitations of the study}

The level of correctness of responses to the survey is questionable. The students weren't not excessive drinkers or smokers. If they were excessive or moderate routine daily drinkers or smokers the acute withdrawal would have caused problems like cognitive problems, disorientation or Disorderliness, hallucinations etc., thankfully there were no moderate routine daily drinkers or smokers and there were also no excessive drinkers or smokers present in the target group.

\section{Reference}

1.van den Hoogen, L. L., Sims, G. P., van Roon, J. A., \& Fritsch-Stork, R. D. (2015). Aging and Systemic Lupus Erythematosus - Immunosenescence and Beyond. Current aging science, 8(2), 158-177. https://doi.org/10.2174/1874609808666150727111904 
2.Chanprapaph, K., M. D., \&amp; Udompanich, S., MD. et al., (2019). Nonscarring alopecia in systemic lupus erythematosus: A cross-sectional study with trichoscopic, histopathologic, and immunopathologic analyses (Vol. 81, Ser. 6). Journal of the American Academy of Dermatology. doi:https://doi.org/10.1016/j.jaad.2019.05.053

3. Chang, K., Yang, S. M., Kim, S. H., Han, K. H., Park, S. J., \& Shin, J. I. (2014). Smoking and rheumatoid arthritis. International journal of molecular sciences, 15(12), 22279-22295. https://doi.org/10.3390/ijms151222279

4. Mamoshina, P., Kochetov, K., Cortese, F. et al. Blood Biochemistry Analysis to Detect Smoking Status and Quantify Accelerated Aging in Smokers. Sci Rep 9, 142 (2019). https://doi.org/10.1038/s41598-018-35704-w

5. Lei, M.-K., Beach, S. R. H., Dogan, M. V. \& Philibert, R. A. A pilot investigation of the impact of smoking cessation on biological age. Am J Addict 26(2), 129-135 (2017).

6. Janssens, Jaak \& Bruckers, Liesbeth \& Joossens, J.V. \& Molenberghs, Geert \& Vinck, Jan \& RENARD, Didier \& Tafforeau, Jean. (2004). Overweight, obesity and beer consumption. Alcohol drinking habits in Belgium and body mass index..

7. Lourenço, S., Oliveira, A. \& Lopes, C. The effect of current and lifetime alcohol consumption on overall and central obesity. Eur J Clin Nutr 66, 813-818 (2012). https://doi.org/10.1038/ejen.2012.20

8. Bachi, K., Sierra, S., Volkow, N. D., Goldstein, R. Z., \& Alia-Klein, N. (2017). Is biological aging accelerated in drug addiction?. Current opinion in behavioral sciences, 13, 34-39. https://doi.org/10.1016/j.cobeha.2016.09.007

9. Carroll, Haley \& Lustyk, M. \& Larimer, Mary. (2015). The relationship between alcohol consumption and menstrual cycle: a review of the literature. Archives of women's mental health. 18. 10.1007/s00737-015-0568-2.

10. Perry, E.C. Inpatient Management of Acute Alcohol Withdrawal Syndrome. CNS Drugs 28, 401-410 (2014). https://doi.org/10.1007/s40263-014-0163-5

11. Vella, Luke \& Cameron-Smith, David. (2010). Alcohol, Athletic Performance and Recovery. Nutrients. 2. 781-9. 10.3390/nu2080781. 
12. Parazzini, F., Tozzi, L., Mezzopane, R., Luchini, L., Marchini, M., \& Fedele, L. (1994). Cigarette Smoking, Alcohol Consumption, and Risk of Primary Dysmenorrhea. Epidemiology, 5(4), 469-472. Retrieved August 4, 2020, from www.jstor.org/stable/3702485 